\title{
Una mirada a la inspección el trabajo. Retos para la ordenación laboral cubana
}

\author{
A look at the inspection work. \\ Challenges for the Cuban labor organization.
}

Uma olbada no trabalbo de inspeção.

Desafios para a organização trabalbista cubana.

Un regard sur l'inspection du travail.

Défis pour l'organisation du travail cubaine

看一下劳动监察.古巴劳工组织面临的挑战

\section{Jennifer Batista Torres ${ }^{1}$ | Universidad de La Habana}

Revista Derechos en Acción ISSN 2525-1678/ e-ISSN 2525-1686

Año 4/No 11 Otoño 2019 (21 marzo a 21 junio), 313-340

DOI: https://doi.org/10.24215/25251678e278

ORCID: https://orcid.org/0000-0002-7945-1079

Recibido: 16/05/2019

Aprobado: 01/06/2019

1 Filiación institucional: Profesora Facultad de Derecho de la Universidad de La Habana.

Dirección postal: 5tab, no 10027 / calle 120 y 146. Apto. 15. Municipio Playa, La Habana, Cuba.

Correo electrónico: jennylex91@gmail.com; jennifer@lex.uh.cu Teléfonos: +53 53540833; +53 72082490

Resumen curricular: Jennifer BATISTA TORRES. (La Habana, 1991). Licenciada en Derecho (Universidad de La Habana, Cuba, 2014) Máster en Derecho Constitucional y Derecho Administrativo (Universidad de La Habana, Cuba, 2018). Estudiante de Doctorado en el Programa de Doctorado en Ciencia Jurídica de la Facultad de Derecho de la Universidad de La Habana, Cuba en cotutela con la Facultad de Derecho de la Universidad de Nanterre, París Francia. Profesor Asistente de Derecho del Trabajo y de Seguridad Social, Facultad de Derecho, Universidad de La Habana. Miembro de la Sociedad Cubana de Derecho Laboral y de Seguridad Social de la Unión Nacional de Juristas de Cuba. Miembro de la Cátedra de Estudios Sindicales de la Universidad de La Habana. Autora de artículos científicos y obras nacionales e internacionales. Ganadora del 4to Concurso Iberoamericano y 6to Nacional de Trabajos Jurídicos, 2019. Ponente en eventos científicos nacionales y extranjeros. Desarrolla investigaciones, fundamentalmente, sobre los derechos laborales, la administración laboral y la inspección del trabajo. 
Resumen: El presente artículo tiene como objetivo fundamental realizar un breve análisis sobre la inspección del trabajo como mecanismo de control del cumplimiento de la legislación de trabajo y de seguridad social por parte de los empleadores. Siendo que nace de la potestad inspectora de la Administración Pública, se utilizan algunos elementos teóricos del Derecho Administrativo en función de explicar cómo se concreta la misma en la Administración Laboral. Teniendo en cuenta dichos particulares, se exponen los retos a los que se enfrenta el ordenamiento jurídico laboral y de seguridad social cubano en materia de la regulación de la inspección del trabajo como una garantía para los trabajadores y como medidor de la eficacia de las políticas públicas laborales en Cuba.

Palabras claves: potestad inspectora, inspección del trabajo, Administración Laboral, Cuba

Abstract: This article has a main objective, which is to carry out a brief analysis of labor inspection as a mechanism to monitor compliance with labor and social security legislation by employers. Being that it is born from the inspection of the Public Administration, some theoretical elements of Administrative Law are used in order to explain how it is concreted in the Labor Administration. Taking into account this ideas, are exposed the challenges faced by the labor legal system and social security in the matter of labor induction as a guarantee for workers and as a measure of the effectiveness of public labor policies in Cuba.

Keywords: inspector power, labor inspection, Labor administration, Cuba

Resumo: 0 presente artigo tem como objetivo principal realizar uma breve análise sobre a inspeção do trabalho como mecanismo de monitoramento do cumprimento da legislação trabalhista e previdenciária pelos empregadores. Sendo que nasce da autoridade fiscalizadora da Administração Pública, se utilizam alguns elementos teóricos do Direito Administrativo para explicar como se concretiza a mesma na Administração Trabalhista. Tendo em conta essas particularidades, estão expostos os desafios que enfrenta o sistema legal trabalhista e de seguridade social cubano em matéria de regulamentação da inspeção do trabalho como uma garantia pelos trabalhadores e como medida de efetividade das políticas públicas trabalhistas em Cuba. 
Palavras-chave: poder inspetor, inspeção do trabalho, Administração Trabalhista, Cuba.

Résumé: L'objectif principal de cet article est de réaliser une brève analyse sur l'inspection du travail en tant que mécanisme qui permet de contrôler l'application par les employeurs des lois du travail et de la sécurité sociale. certains éléments théoriques de la loi administrative sont utilisés afin d'expliquer comment elle est concrétisée dans l'administration du travail, étant donné qu'il est issu de l'autorité de contrôle de l'administration publique. Compte tenu de ces particularités, les défis auxquels le système juridique du travail et la sécurité sociale cubaine sont confrontés en matière de réglementation de l'inspection du travail, en tant que garantie des travailleurs et mesure de l'efficacité des politiques publiques á Cuba, sont exposés.

Mot-clés: autorité d'inspection, inspection du travail, Administration du travail, Cuba.

摘要: 本文的主要目的是对劳动监察作为监督雇主遵守劳动和社会 保障立法的机制进行简要分析. 由于它是由公共行政部门的检查机 构产生的, 因此使用行政法的一些理论要素来解释它在劳动行政管 理中是如何具体化的. 考虑到这些细节, 劳动法律制度和古巴社会保 障在劳动监察管理作为工人保障和衡量公共政策有效性方面面临 的挑战暴露无遗.在古巴就业。

关键字: 检查员的权力, 劳动监察, 劳动行政管理, 古巴

\section{Notas iniciales}

Desde tiempos inmemoriales el hombre necesitó trabajar. Con el trabajo logró obtener medios de subsistencia por lo cual éste se convirtió en el pilar fundamental para la evolución de la especie humana. La segunda mitad del siglo XVIII fue un momento crucial para el trabajo con la Revolución Industrial. Con ella se transformaron las estructuras sociales y las formas tradicionales de las prestaciones de laborales (Grisolia, 1999). Es, en definitiva, con el entronizamiento del capitalismo, lo que permitió la transformación de las relaciones sociales (De 
Buen, 1984) que se expresaron en las relaciones "obreroempleador». Así, los trabajadores comenzaron a prestar sus servicios personales, pero en inhumanas condiciones, lo cual conllevó a un enfrentamiento entre éstos y sus empleadores. Entonces, ante las precarias condiciones de trabajo y con la intención de proclamar derechos mínimos surge el Derecho del Trabajo (Abramovich y Courtis, S.f). La nueva rama procuró la protección a los trabajadores que laboraran en condiciones de ajenidad, subordinación y dependencia (Diéguez, 1995).

De tal suerte, se evidenció la intervención (Hernaiz Márquez, 1981; Alonso Olea, 1981) del Estado en la regulación jurídica de las nuevas relaciones en torno al trabajo, proyectándose además hacia el control del cumplimento de la normativa reguladora de dichas relaciones. La referida intervención no fue igual ante todos los supuestos, y se emplearon en ello diversas técnicas jurídicas (Blasco Pellicer y García Rubio, 2001). Pero para el caso de las relaciones laborales, el Estado creó servicios administrativos -Administración Laboral- destinados a intervenir en este orden; especialmente a través de la actividad de inspección "del trabajo", que derivó de la potestad inspectora del Estado.

La inspección del trabajo es un mecanismo de control del cumplimiento de las disposiciones laborales en el marco de las relaciones jurídicas de trabajo. Lleva implícita una titularidad pública y como actividad puede ser realizada por el Estado o por entes diferentes, incluso privados (Bermejo Vera, 1998).

De resultas que en la inspección del trabajo tiene el Estado un medio para garantizar la eficacia normativa (Alonso Olea y Casas Baamonde, 1995) en estas cuestiones. Asimismo, la inspección del trabajo es una vía de defensa de los derechos de los trabajadores. (Vázquez Vialard, 1999)

Si se mira algo hacia el pasado, se puede ver que la inspección del trabajo tuvo sus antecedentes más remotos en el siglo XVIII, con la figura de los veedores de las corporaciones gremiales, los cuales vigilaban el cumplimiento de las normativas de los oficios (De Aguinaga Tellería, 1952). Durante el siglo XIX, 
dicha inspección estaba fundamentalmente dirigida a verificar el cumplimiento de las normas relativas a la higiene industrial. (Pig, 1922) Con posterioridad se establecieron otros servicios de inspección, ampliando las materias a controlar (Viamontes Guilbeaux, 2007). De todo este íter nació la inspección del trabajo como actividad pública, cuyo fin primordial, gradualmente adquirido, fue el de velar por la correcta aplicación de las leyes laborales ${ }^{2}$.

La inspección del trabajo se reconoció inicialmente en el ámbito nacional, pero en 1919, con la fundación de la Organización Internacional del Trabajo (OIT), se internacionalizó ${ }^{3}$. De esta manera, se colocó como un instrumento fundamental dentro de la Administración del Trabajo y ha sido objeto de estudios y propuestas (OIT, 2009; OIT, 2010; OIT, 2011). Igualmente, la OIT adoptó los Convenios 81, 129 (ambos prioritarios) y 178; el Protocolo 81; así como las Recomendaciones 20, 28, 81, 133 y 185 relativas todas a la inspección del trabajo. Por otro lado, las corrientes político jurídicas actuales (impulsadas por la propia OIT) colocan a la inspección del trabajo como un indicador fundamental del trabajo decente (Casale y Fasani, 2013), al ser herramienta eficaz y segura para controlar el cumplimiento de la legislación de trabajo.

En la actualidad con el fenómeno de la globalización, con el fortalecimiento del capitalismo y con las continuas crisis económicas, se hace indispensable fortalecer la protección a la clase obrera. La Administración del Trabajo, a través de esta

\footnotetext{
2 Siendo así, posee puntos de contacto con todas las categorías e instituciones del Derecho del Trabajo, teniendo que controlar que se cumpla lo dispuesto en materia de contratación laboral, salario, jornada de trabajo, descansos y vacaciones, seguridad y salud, accidentes y enfermedades profesionales, trabajo infantil, política de empleo, disciplina de trabajo y solución de conflictos.

3 Según el Tratado de Versalles cada Estado debería organizar un servicio de inspección a fin de asegurar la aplicación de las leyes y reglamentos para la protección de los trabajadores Cfr. Artículo 427. 9 del Tratado de Versalles de 28 de junio de 1919, depositario: República Francesa disponible en http://www.cervantesvirtual.com/descargaPdf/tratadode-versalles/, consultado el 31 de mayo de 2017.
} 
inspección, posee una vía necesaria para proveer a esa protección donde quiera que existan relaciones jurídicas laborales. Al presente, la inspección del trabajo está sujeta a diversos retos en tanto el mundo del trabajo es cambiante, diverso e imprevisible. Entre estos retos se encuentran la aparición de requisitos específicos técnicos, de nuevos riesgos para la salud, el uso de las nuevas tecnologías (TICs), el envejecimiento poblacional, las tasas elevadas de empleo de mujeres y de desempleo de jóvenes, las migraciones calificadas, las nuevas formas de organización del trabajo y las pretensiones de disminuir los ámbitos ganados en favor de los derechos de los trabajadores. Todos ellos hacen que la inspección del trabajo deba modificar su concepción clásica para llevar a cabo su cometido y ajustarse a las nuevas realidades que se han ido revelando.

Cuba no se encuentra ajena a estos fenómenos por lo cual se enfrenta a varios retos en medio de una actualización de su modelo económico, social y jurídico.

\section{Naturaleza jurídica de la inspección del trabajo}

La inspección del trabajo puede ser definida como aquella potestad que tiene la Administración de Trabajo y que lleva a cabo con el fin de controlar el cumplimiento de la legislación laboral y de seguridad social. Sin embargo, existen diversos criterios sobre su naturaleza jurídica que influyen directamente en una concepción acabada.

En este sentido, autores como Bermejo Vera (2015, p. 63) afirman que: "se habla de actividad, función o potestad de inspección, sin excesiva precisión en este momento, para identificar unas actuaciones, más o menos genéricamente previstas en el ordenamiento jurídico, que habilitan a las Administraciones públicas para llevar a cabo funciones de comprobación o constatación del cumplimiento de la normativa vigente, en su sentido más amplio, esto es, incluidas muy especialmente las condiciones y requisitos de orden técnico, consecuencia inherente de la imposición que a determinadas personas, 
actividades, instalaciones, objetos y productos hacen ciertas normas jurídicas. El motivo principal de la dotación y ejercicio de estas funciones de inspección y control es, en cualquier plano, la seguridad".

En este sentido, lo más trascendente es que "ya sea considerada potestad, actividad o función" se trata de aludir a las acciones que realice la Administración Pública para constatar el cumplimiento de la normativa, especialmente la laboral en este caso.

Otros como Rebollo Puig plantean que la inspección es una actividad auxiliar "porque siempre tiene por finalidad detectar hechos que deben dar origen al ejercicio de otras potestades administrativas: no solo la sancionadora, como simplistamente puede pensarse, sino a otras muchas de intervención de entidades de crédito, de reintegro de subvenciones, de restablecimiento de la legalidad, etc. Aun así, ella misma cumple por sí sola una función de prevención general que refuerza la observancia de los deberes. Y, en cualquier caso, aunque auxiliar de otras, se presenta formalmente al margen de esas otras". (2009, p. 55).

Y en la doctrina cubana, influenciada a su vez por la soviética (Vasilenkov, 1989, p 159), la inspección se entiende como la facultad "en el sentido de potestad" que posee un órgano administrativo para examinar los actos materiales y las operaciones preparatorias y ejecutivas de los actos de decisión que tienen a su cargo los trabajadores que le están subordinados. Nunca se proyecta sobre los actos productores de efectos jurídicos (Garcini Guerra, 1978, p. 169).

A partir de esto la inspección posee determinadas características. Tales estarán definidas por la concepción como potestad, como función pública o como actividad. Así, como potestad es una forma de intervención de la Administración pública en la cual no cabe la discrecionalidad. La misma debe de ajustarse a un canon de objetividad, eficiencia, transparencia y responsabilidad, que permitan asegurar, desde la planificación y control de su ejercicio, la debida neutralidad, probidad y cumplimiento 
estricto de la juridicidad por parte del inspector. (Sánchez Ramírez y Antúnez Ramírez, 2018, p. 359). Esta se materializa en el reconocimiento de competencias a los diversos órganos especializados en esta materia.

Como actividad se refiere precisamente a la posibilidad de realizar dichas actividades de investigación y constatación en función de la actividad de policía o de control. Y, como función pública se relaciona con todo lo referido al ejercicio de tal por los inspectores designados al efecto.

En esencia, la naturaleza jurídica de la inspección del trabajo está enriquecida por ser una tríada que propende a mayor seguridad jurídica. En el caso específico de la inspección del trabajo, tal se traduce en verificar y promover la protección de los trabajadores frente al ejercicio arbitrario e ilegal del poder de dirección de los empleadores. Sobre todo, en la actualidad, tal particular adquiere especial relevancia con los procesos de globalización neoliberal, nuevas formas de empleo y el uso de las Tics para la realización del trabajo.

\section{Principios de la inspección del trabajo}

En este caso, los principios no quedan definidos en ningún instrumento jurídico internacional. Sin embargo, siendo la inspección una actividad-función-potestad de la Administración Publicación es válido la identificación de los mismos partiendo de aquellos que informan todo su actuar.

En primer lugar, el principio de legalidad, referido a que la Administración Pública solo podrá inspeccionar en la medida que ostente la debida habilitación legal para hacerlo, independiente de la concreta técnica de atribución que se haya utilizado al respecto, tal y como afirma Leal Vásquez (2015a, p. 94). De esta forma, dicho principio se convierte en el sostén estructural de dicha potestad en tanto responde a la verificación de lo regulado y a su vez, asegura la sujeción al procedimiento establecido legalmente para ello. En el caso concreto de la inspección del trabajo este principio se manifiesta en el reconocimiento de tal 
potestad a los entes administrativos laborales y de las prerrogativas que posee el inspector y en los procedimientos que debe realizar; así como la definición de las materias a inspeccionar.

Derivado del principio de legalidad se encuentra el de objetividad. Tal se refiere a la interpretación que debe realizar el inspector no solo de las circunstancias observadas durante su actividad sino de la toma de decisiones atendiendo a la racionalidad sin que medie interés personal o subjetividad de modo que la interpretación de la norma no sea ajena a la voluntad del legislador (García Costa, 2011, p. 34).

Igualmente, este principio se vincula al ámbito subjetivo de la función inspectora debido al valor probatorio de sus informes y actas de inspección en los procedimientos administrativos (Míguez, 2011, pp. 119 y ss.) "y judiciales especialmente en la materia laboral". Además, el principio impone la actuación intachable del inspector, ajeno a favoritismos y a parcializaciones de cualquier índole. Por ello esta función debe ser desarrollada de modo independiente; es decir sin predisposiciones o indicaciones de superiores jerárquicos de fuerzas políticas o administrativas externas que comprometan la actividad. En resumen, el inspector se obliga solo al estricto deber de comprobación de compatibilidad de la legalidad con la actividad inspeccionada sin modulaciones internas o externas de juicios o convicciones personales o externas (Cano, 2013, p 226; Matilla, 2017, pp. 95-97).

Todo esto se traduce en la inspección de trabajo precisamente en que la labor de los inspectores de trabajo como funcionarios públicos los independicen de los cambios de gobierno y de cualquier influencia exterior indebida. (Artículo $6^{\circ}$ Convenio 81) y en el hecho de que "siempre que sea compatible con la práctica administrativa del Miembro, la inspección del trabajo deberá estar bajo la vigilancia y control de una autoridad central" (Artículo $4^{\circ}$ Convenio 81).

Otro de los principios aplicables a la actividad de inspección es el de eficiencia referido a la evaluación de las formas en 
las que se emplean los recursos públicos en función de que su uso resulte razonable a los fines de la satisfacción de las necesidades de los ciudadanos y de la propia Administración. De este modo se refiere a la necesidad de la consecución de los fines públicos a través del gasto correspondiente a esa consecución. Matilla (2017, p. 87) Aplicado a la inspección del trabajo tiene idéntica connotación, la Administración Laboral debe realizar su cometido con el uso de los recursos disponibles a su alcance, que deben ser suficientes tal y como disponen los artículos 10 y 11 del Convenio 81.

Por su parte, el principio de proporcionalidad (Stiftung, 2003) referido al alcance de la potestad inspectora en el justo balance de ponderación entre la ejecución de la propia actividad, las circunstancias concurrentes en el momento de la inspección y las medidas aplicadas como resultado de la misma de modo que no resulte inoperante, inactiva (Rivero, 2007, p. 162) ni fuera de los márgenes legales establecidos; se manifiesta en la materia laboral precisamente en que la realización de la inspección debe tener siempre el fin de verificar el cumplimiento de las disposiciones laborales en función de la protección del trabajador y en defensa de sus derechos, atendiendo, no solo a las circunstancias existentes en el momento, sino a las condiciones reales "principio de primacía de la realidad" del medio laboral "entendido este en sentido amplio". Del resultado de la misma deben derivar medidas para el cumplimiento de las obligaciones del empleador que permiten el goce de los derechos a los trabajadores en el marco del trabajo; el perfeccionamiento de las condiciones del medio laboral, la retrospección al estado normal de condiciones dignas en el trabajo y de trabajo. Sin embargo, ninguna de estas medidas puede representar una carga para el trabajador y menos colocarlo en una posición de deber frente a su empleador.

Por ello, el principio de proporcionalidad en la inspección del trabajo posee caracteres especiales en tanto su cumplimiento deriva de la actuación de los inspectores en la justa medida 
atendiendo el principio de eficiencia, legalidad y protectorio del trabajador.

\section{Sujetos de la inspección del trabajo}

Es imprescindible referirnos al elemento subjetivo de la inspección. Como ya se explicó la inspección del trabajo es la única institución del Derecho Laboral cuyo sujeto directo no es el trabajador, aunque en definitiva propenda a la protección del mismo. Es decir, la inspección del trabajo está dirigida a la protección de los trabajadores a través del control que se realice a los empleadores. Bajo ninguna circunstancia, la actividad inspectora estará dirigida a los trabajadores.

En este sentido, es importante señalar además que los Convenios internacionales en la materia refieren este particular haciendo alusión al ámbito espacial de la inspección. De esta manera, los sujetos inspeccionados serán los empleadores de los establecimientos industriales y comerciales (C81-1947), a los establecimientos no comerciales (P81-1995), aquellos de las entidades en territorios metropolitanos (C85-1947), a las empresas agrícolas (C129-1969) y a todos los buques dedicados a la navegación marítima con fines de transporte de mercancías o de pasajeros o empleado en cualquier otro uso comercial (C178-1996). Ello se traduce en que más allá de que lo que realmente trasciende para la inspección es precisamente el ámbito en que se realiza el trabajo, será la figura del sujeto empleador el obligado al cumplimiento de las normativas jurídicas relativas a los derechos laborales y de seguridad social.

De resultas, otra figura también personifica esta actividad de inspección: el inspector de trabajo. Este sujeto es un funcionario público por definición (Artículo 6 del C81-1947). Quien, además, debe tener un régimen de empleo que le garantice estabilidad y lo independice de cualquier cambio de gobierno y de cualquier influencia exterior indebida. A su vez el C178-1996 (artículo $7^{\circ}$ ) reafirma la definición exponiendo que el inspector es todo funcionario u otra categoría de empleados públicos 
encargados de la inspección de cualquier aspecto de las condiciones de vida y de trabajo (...), así como a toda persona debidamente acreditada que realice una labor de inspección para una institución u organización facultada por la autoridad central de coordinación (...). Por ello, es totalmente aplicable a dichos sujetos las cuestiones teóricas sobre la función pública y el ejercicio de la misma.

Igualmente, es importante agregar, de manera muy somera que los inspectores de trabajo deben tener identificados en un cuerpo jurídico específico los requisitos de idoneidad para ejercer como tal, las atribuciones, facultades y prohibiciones. Tales están definidos explícitamente en los diferentes Convenios de la OIT sobre la materia.

\section{Funciones y organización del sistema de inspección}

El sistema de inspección del trabajo se refiere al conjunto de estructuras interrelacionadas entre sí que llevan a cabo la actividad de inspección. Su organización depende de cada ordenamiento jurídico concreto; sin embargo, el C-81 propone que debe estar bajo la vigilancia y el control de una autoridad central. Además, el sistema de inspección puede estar conformado por un grupo de inspectores individualmente asignados o existir oficinas locales en dependencia de la distribución político administrativa de cada Estado. Lo cierto es que la organización de los sistemas de inspección debe garantizar el cumplimiento y control de la legislación de trabajo y de seguridad social por parte de los empleadores.

Dicho sistema, posee un conjunto de funciones que pueden identificarse desde la teoría general de la inspección a través de las potestades que la Administración Laboral les otorga a los inspectores.

a) Potestad de acceso a lugares privados. No solo a los abiertos al público, que no plantean problemas, sino a locales cerrados, a dependencias de uso privado como oficinas, 
instalaciones y su entorno, medios de transporte o buques. De ordinario, se trata de entrar en los locales que dependen del inspeccionado o, en su caso, de las personas a su servicio. Pero a veces se requiere y se permite el acceso a locales de otros sujetos; por ejemplo, a un local contiguo a aquel del que emana un ruido para hacer las mediciones necesarias (Rebollo Puig, 2004, p. 309).

Esta potestad incluye o hasta se identifica con la de observación directa de cuantas cosas (materias primas o productos terminados, máquinas, animales...) o personas allí se encuentren.

b) Potestad de examen de la documentación, y de obtener copias. A veces se configura como facultad de hacer de la Administración que le posibilita el acceso directo a los documentos de forma que el administrado solo tiene que dejar actuar, mientras que en otros casos se presenta como una facultad de ordenar la exhibición o remisión de documentos que comporta para el destinatario un deber de hacer. Puede afectar al inspeccionado o, en su caso, a terceros por sus relaciones con el inspeccionado. No en todos los casos se establece con carácter general sino acotando más o menos los documentos que pueden ser objeto de examen. (Rebollo Puig, 2004, p. 310).

c) Potestad de recabar información y exigir respuestas a las preguntas que se formulen, tanto del inspeccionado como, en su caso, si está previsto, de su personal y hasta de terceros (suministradores y prestadores de servicios al inspeccionado, clientes y usuarios del inspeccionado, etc.). (Rebollo Puig, 2004, p. 310).

d) Potestad de tomar muestras de diferentes bienes para análisis, incluso de bienes de propiedad de persona distinta del inspeccionado. (Rebollo Puig, 2004, p. 311).

e) Potestad de recabar de otros órganos administrativos información o documentación. No se trata solo de que en virtud del deber general de colaboración "las Administraciones Públicas podrán solicitar cuantos datos, documentos o medios 
probatorios se hallen a disposición del ente al que se dirijan" y de que la que reciba tal petición deba "facilitar a las otras Administraciones la información que precisen sobre la actividad que desarrollen en el ejercicio de sus propias competencias". (Rebollo Puig, 2004, p. 311)

Estas potestades pueden estar completadas con la de adoptar medidas provisionales, no ya para impedir que se siga cometiendo una actuación ilícita o produzca sus efectos lesivos, que ya es cuestión ajena a la función de inspección, sino para asegurar su propia investigación. (Manuel Rebollo Puig, 2013, p. 64).

Este conjunto de funciones se manifiesta específicamente en la inspección del trabajo según lo que establece el C81- 1947.

El artículo 3 de dicho convenio dispone que el sistema de inspección estará encargado de:

a) velar por el cumplimiento de las disposiciones legales relativas a las condiciones de trabajo y a la protección de los trabajadores en el ejercicio de su profesión, tales como las disposiciones sobre horas de trabajo, salarios, seguridad, higiene y bienestar, empleo de menores y demás disposiciones afines, en la medida en que los inspectores del trabajo estén encargados de velar por el cumplimiento de dichas disposiciones;

b) facilitar información técnica y asesorar a los empleadores y a los trabajadores sobre la manera más efectiva de cumplir las disposiciones legales;

c) poner en conocimiento de la autoridad competente las deficiencias o los abusos que no estén específicamente cubiertos por las disposiciones legales existentes.

Igualmente se dispone que ninguna otra función que se encomiende a los inspectores del trabajo deberá entorpecer el cumplimiento efectivo de sus funciones principales o perjudicar, en manera alguna, la autoridad e imparcialidad que los inspectores necesitan en sus relaciones con los empleadores y los trabajadores. (Artículo 3.2 C81-1947). 
A su vez, los inspectores del trabajo tendrán la facultad discrecional de advertir y de aconsejar, en vez de iniciar o recomendar un procedimiento. (Artículo 17 C81.1947).

En el caso específico de la inspección en la agricultura las funciones de los inspectores serán (artículo 6 C129-1969):

a) velar por el cumplimiento de las disposiciones legales relativas a las condiciones de trabajo y a la protección de los trabajadores en el ejercicio de su profesión, tales como las disposiciones sobre horas de trabajo, salarios, descanso semanal y vacaciones; seguridad, higiene y bienestar; empleo de mujeres y menores, y demás disposiciones afines, en la medida en que los inspectores del trabajo estén encargados de velar por el cumplimiento de dichas disposiciones;

b) proporcionar información técnica y asesorar a los empleadores y a los trabajadores sobre la manera más efectiva de cumplir las disposiciones legales;

c) poner en conocimiento de la autoridad competente los defectos o los abusos que no estén específicamente cubiertos por las disposiciones legales existentes, y someter a ella proposiciones para mejorar la legislación.

\section{Retos para la ordenación laboral cubana}

Cuba no se encuentra hoy ajena a un escenario donde, con sus particularidades, la inspección del trabajo tiene relevancia per se. En nuestra realidad político-jurídica, la protección de las relaciones laborales adquiere un papel esencial atendiendo sobre todo a los fundamentos constitucionales del Estado cubano ${ }^{4}$, a la importancia del trabajo ${ }^{5}$ y a los principios sobre los cuales se

\footnotetext{
4 Artículo 1: Cuba es un Estado socialista de derecho y justicia social, democrático, independiente y soberano, organizado con todos y para el bien de todos como república unitaria e indivisible, fundada en el trabajo, la dignidad, el humanismo y la ética de sus ciudadanos para el disfrute de la libertad, la equidad, la igualdad, la solidaridad, el bienestar y la prosperidad individual y colectiva.

5 Vid. Artículos 1, 20, 31, 42, 43 y 64 y ss. de la Constitución cubana de 2019 en Gaceta Oficial Extraordinaria No. 5 de 10 de abril de 2019.
} 
erige el sistema laboral ${ }^{6}$. Así, la calidad en la ordenación y en la ejecución de la inspección del trabajo en Cuba se convierte en un tema vital.

En Cuba, no se han esclarecido los antecedentes históricojurídicos de la inspección del trabajo; de ahí que exista dificultad para comprender indubitadamente el íter evolutivo de ese fenómeno jurídico.

Sin descartar la mirada al período previo al nacimiento de Cuba como Estado, es sin dudas a partir de esta etapa en el siglo XX que puede ser más interesante el análisis histórico. En la etapa republicana prerrevolucionaria, la Ley Orgánica del Poder Ejecutivo de 1909, instituyó la Secretaría de Agricultura, Comercio y Trabajo y dentro de sus facultades estableció la alta inspección en los establecimientos industriales ${ }^{7}$. Esto permitió vislumbrar el nacimiento de una administración especial en la materia y que tuvo su punto cumbre con el Decreto Presidencial No. 2142 de $1933,{ }^{8}$ el cual creó la Secretaría de Trabajo. El Decreto No. $2335^{9}$ del propio año 1933, definió las materias de competencia de dicha secretaría y la Ley No. $91^{10}$ del año siguiente estableció sus funciones y estructura ${ }^{11}$. Pero no se puede afirmar que existió ninguna disposición jurídica orgánica y sistematizada que regulara la inspección del trabajo durante esta época ${ }^{12}$. No obstante,

6 Vid. Artículo 2 de la Ley No. 116 Código de Trabajo de 20 de diciembre de 2013 en Gaceta Oficial Extraordinaria No. 29 de 17 de junio de 2014.

7 Cfr. Artículos 247 y 248 del Decreto No. 78 del Gobernador Provisional en Cuba en Gaceta Oficial de 12 de enero de 1909.

8 Cfr.Decreto No. 2142 de 13 de octubre de 1933 en Gaceta Oficial de 16 de octubre de 1933.

9 Cfr.Decreto No. 2355 de 25 de octubre de 1933 en Gaceta Oficial de 26 de octubre de 1933.

10 Cfr.Ley No. 91 de 12 de abril de 1935 en Gaceta Oficial Extraordinaria de 16 de abril de 1935

11 Vid. Méndez Peñate, Rodolfo: Curso de legislación obrera, 2do Curso, T.: I, Imprenta Modas Magazine, La Habana, 1953, pp. 293 y ss.

12 Las normativas más cercanas a la materia fueron las leyes de accidentes de trabajo (1916, 1933) e higiene del trabajo (1948). 
un hecho a destacar fue el reconocimiento de la inspección del trabajo en el artículo $85^{13}$ de la Constitución de 1940, lo que encierra un momento importante en su régimen jurídico, al menos en lo formal, porque con ese reconocimiento adquiere rango constitucional. Para 1954, Cuba ratificó el Convenio 81 (1947), que dispuso la creación de sistemas de inspección en establecimientos industriales.

Al triunfo revolucionario en 1959, la Ley Fundamental ${ }^{14}$ conservó en el artículo 85 la referencia a la inspección del trabajo. En 1960, en Cuba existía una División de Revisión e Inspección Social ${ }^{15}$ y en 1972 se creó la Dirección de Inspección Nacional del Trabajo.

Por su parte, la Constitución de 1976, a diferencia de la de 1940, no contuvo reconocimiento expreso sobre la inspección del trabajo; aun cuando se contienen en ese texto constitucional esenciales referencias al trabajo, incluso enraizado en la esencia misma de nuestro Estado socialista. La vigente Constitución del año 2019 mantiene idéntica omisión, aunque logra enfatizar la trascendencia del trabajo y de los trabajadores para la nación.

El funcionamiento de la inspección fue regulado inicialmente, por los Decretos No. 100 de $1982^{16}$, No. 116 de $1983^{17}$. Posteriormente, al promulgarse la Ley No. 49 de 1984, Código

13 Artículo 85.- A fin de asegurar el cumplimiento de la legislación social, el Estado proveerá a la vigilancia e inspección de las empresas. Cfr. Ley de 1 ro de julio de 1940 (Constitución de la República de Cuba) en Gaceta Oficial No. 464 de 8 de julio de 1940.

14 Cfr. Ley Fundamental de 7 de febrero de 1959 en Gaceta Oficial Especial No. de 8 de febrero de 1959.

15 Cfr. Ley No. 696 de 22 de enero de 1960, de la estructuración orgánica del Ministerio de Trabajo en Gaceta Oficial Extraordinaria No. 2 de 25 de enero de 1960.

16 Cfr. Decreto No. 100 Reglamento General de la Inspección Estatal en Gaceta Oficial Ordinaria No. 14 de 10 de febrero de 1984.

17 Cfr. Decreto No. 116 Reglamento para la inspección sindical de protección e higiene del trabajo en Gaceta Oficial Ordinaria No. 54 de 20 de julio de 1983. Este tipo de inspección es un complemento a la inspección del trabajo. 
de Trabajo $^{18}$ la inspección del trabajo fue ordenada en los artículos 296 y siguientes.

Ya en los años '90 las relaciones laborales iniciaron un proceso de redimensionamiento lo que dio al traste el desfase de dichas normas en cuanto a relaciones laborales e inspección del trabajo.

En el 2002, respondiendo a las nuevas circunstancias económicas, políticas y sociales, se constituyó la Oficina Nacional de Inspección del Trabajo (ONIT) ${ }^{19}$ como entidad administrativa adscripta al Ministerio de Trabajo, con la misión de comprobar el cumplimiento de la política en materia de trabajo y seguridad social. Tal institución tiene oficinas territoriales que cumplen la función de inspección en las entidades que tiene bajo su cargo.

Alrededor de esto, encontramos el primer reto que deriva de la interrogante de la incidencia de la estructura administrativa en los niveles territoriales que llevan a cabo la inspección del trabajo. Igualmente, hace pensar en la naturaleza de la inspección: administrativa, laboral o mixta.

En el año 2007 vino a completar la regulación de esta institución la Resolución No. 20 de $2007^{20}$ la cual estableció los fines, el objeto y el contenido de la actividad de inspección nacional del trabajo.

Actualmente, la inspección del trabajo queda regulada por los Decretos No. 100 de 1982 y 116 de 1983 y por el vigente Código de Trabajo (CT) ${ }^{21}$ y su Reglamento ${ }^{22}$.

18 Cfr. Ley No. 49 Código de Trabajo en Gaceta Oficial Ordinaria No. 93 de 28 de diciembre de 1984.

19 Mediante Acuerdo 4085 de 2001 y la Resolución 590 del Ministerio de Economía y Planificación (MEP), del propio año.

20 Cfr. Resolución No. 20 Reglamento del Sistema de Inspección Nacional del Trabajo de 6 de abril de 2007 en Gaceta Oficial Extraordinaria No. 18 de 18 de abril de 2007.

21 Cfr. Artículos 190 y ss. del CT.

22 Cfr. Artículos 222 y ss. del Decreto No. 326 Reglamento del Código de Trabajo de 12 de junio de 2014 en Gaceta Oficial Extraordinaria No. 29 de 17 de junio de 2014. 
El Código de Trabajo, en sus artículos 188 y siguientes regula esta institución bajo el capítulo de las autoridades de trabajo. El artículo 190 realiza una definición de la inspección del trabajo que se pudiera denominar funcional al establecer que tal institución controla el cumplimiento de la legislación de trabajo, de seguridad social y dispone o aplica las medidas legalmente establecidas. Tal descripción deja entrever como la inspección del trabajo en Cuba deriva de la potestad inspectora de la Administración Pública y de la potestad sancionadora en tanto no solo controla el cumplimiento de la legislación laboral, sino que también, en correspondencia con lo establecido, dispone y aplica directamente medidas para que se cumplan las disposiciones laborales y de seguridad social vulneradas.

El articulado del Código determina también las autoridades con facultad para inspeccionar y el ámbito de fiscalización. Así, el Ministerio de Trabajo y Seguridad Social (MTSS) controlará lo relativo a la legislación de trabajo y seguridad social; el Ministerio de Salud Pública (MINSAP) las cuestiones de medicina e higiene del trabajo; el Ministerio del Interior (MININT) lo concerniente a la protección contra incendios, explosivos y sustancias peligrosas. Por su parte, el Ministerio de Ciencia, Tecnología y Medio Ambiente (CITMA) dirige su fiscalización a las cuestiones relacionadas con las sustancias biológicas, radiológicas y al medio ambiente y el resto de los Organismos de la Administración Central del Estado (OACE), entidades nacionales y los Consejos de Administración también podrán crear sus sistemas de inspección y llevar a cabo dicha función.

Y de aquí deriva otro reto al que se enfrenta la inspección del trabajo: la atomización de la actividad de inspección en varias normas jurídicas y que dependen de la competencia de los $\mathrm{OACE}^{23}$ que rigen la actividad de inspección. Además, los sindicatos complementan dicha actividad en tanto tienen

23 Cfr. Artículo 191 de la Ley No. 116 Código de Trabajo de 20 de diciembre de 2013 en Gaceta Oficial Extraordinaria No. 29 de 17 de junio de 2014. 
reconocida la facultad para inspeccionar en relación con la seguridad y salud en el trabajo ${ }^{24}$. El Decreto 116 dispone los particulares sobre dicha inspección sindical y alude a los requisitos para ser inspector, así como los de la inspección técnica profesional y de las comisiones de protección e higiene del trabajo.

Atinadamente continúa el Código refiriendo que la Oficina Nacional de Inspección del Trabajo es quien ejecuta dicha función, así como sus filiales en los territorios.

En cuanto a los sujetos inspeccionados son todos los empleadores radicados en el territorio nacional, y otros que la ley determine. En este sentido existe una interrogante que parte de la idea de los trabajadores por cuenta propia titulares de licencia; que en buena lid comparten el doble estatuto de empleador-trabajador ¿Son sujetos de inspección? Sí, de acuerdo al artículo 9 inciso b) del CT.

El CT concluye en su artículo 193 refiriendo que los resultados de la inspección deben ser informados al empleador, a la organización sindical correspondiente y a los trabajadores. Dicho informe podrá ser utilizado como prueba documental en cualquier procedimiento.

Por su parte el Decreto 326 Reglamento del CT, en sus artículos 222 y siguientes regula que se considera como infracciones cometidas por el empleador. A estos efectos lo son la inobservancia o el incumplimiento de las normas jurídicas o acuerdos de los órganos superiores del Estado y el Gobierno y de las disposiciones o decisiones sobre trabajo y seguridad social emitidas por los órganos competentes y las autoridades facultadas.

El artículo 223 establece como regla que la acción para exigir responsabilidad prescribe cuando la infracción y sus efectos están eliminados al momento de la inspección. Igualmente, deja

24 Vid. Decreto No. 116 Reglamento para la inspección sindical de protección e higiene del trabajo en Gaceta Oficial Ordinaria No. 54 de 20 de julio de 1983. 
ver una contradicción. Dispone el segundo párrafo que cuando de los hechos considerados como infracciones puede derivarse la exigencia de responsabilidad penal, el inspector lo informa a la autoridad que ordenó la realización de la inspección, a los fines de formular la denuncia ante la autoridad correspondiente. De este enunciado puede interpretarse que solamente podrán solicitar la inspección autoridades, sin especificar ningún otro particular. No deja abierta la posibilidad de que la organización sindical o el propio trabajador puedan requerir la inspección. Por otro lado, implica que el inspector informe a la autoridad que ordenó la inspección para que denuncie ¿cuál es esa autoridad? Esto lo aclara el artículo 235 del propio cuerpo legal al explicar que la denuncia a las autoridades penales se presenta por escrito fundamentado del Director General de la Oficina Nacional de Inspección del Trabajo o los directores de las filiales territoriales según corresponda.

A continuación, el Reglamento establece por materia las infracciones en las que puede incurrir el empleador: infracciones de los derechos fundamentales en el empleo y la contratación; en la protección a la trabajadora; en el régimen de trabajo y descanso; en el salario; en la seguridad y salud en el trabajo; y en la seguridad social. Así como un artículo 230, artículo saco, que, en buena lid, es el que establece las infracciones en materia de cumplimiento de la legislación laboral y de seguridad social.

Así mismo, el artículo 231 establece que la autoridad que inspecciona podrá aplicar directamente tres tipos de medidas:

a- Obligación de hacer: con un apercibimiento por escrito al infractor para que cesen las causas y los efectos de la infracción.

b- Clausura, paralización inmediata de equipos, maquinarias, cierre de locales, etc.

c- Multa a personas jurídicas y naturales, cubanas y extranjeras del sector no estatal.

Sin embargo, la autoridad inspectora debe solicitar la aplicación de otras medidas ante la comisión de infracciones. 
a- Medidas en el orden administrativo o disciplinario en correspondencia con la gravedad de los hechos.

b- La suspensión temporal o definitiva de licencias y permisos otorgados al empleador.

c- El inicio de un proceso penal a los presuntos responsables de accidentes mortales, a las autoridades facultadas.

$\mathrm{Y}$ en este último inciso pudiera valorarse que no sea solo ante la ocurrencia de accidentes mortales, sino ante otras conductas, nacidas en el ámbito de las relaciones laborales y que el Código Penal proteja bajo bienes jurídicos diferentes o que sencillamente, la política penal así lo decida. V. gratia. asuntos relativos al trabajo de los menores, acoso laboral, falsificación de documentos relativos a las relaciones laborales, discriminación, trabajo forzoso, etc.

El Reglamento deja entrever en los artículos 223 y ss. algunos particulares. En primer lugar, la existencia de un acta de inspección donde se esclarecerá la disposición infringida, la medida que se aplica, el término de su cumplimiento, el carácter recurrible, la autoridad para conocer y resolver el recurso y el término para ello.

Frente a los resultados de la inspección, el empleador controlado puede interponer un recurso de apelación a la instancia a la cual se subordine el inspector que realizó el control; lo cual no interrumpe el cumplimiento de la medida. En un término de 10 días hábiles, la autoridad facultada debe resolver el recurso mediante Resolución contra la cual no cabe recurso alguno. En este sentido, sería interesante valorar qué sucede cuando es el trabajador quien se encuentra inconforme con el resultado de la inspección. ¿No se verá acaso disminuida su protección que, en definitiva, es a lo que propende la inspección del trabajo?

En consecuencia, esta regulación es aún insuficiente. Se extraña del texto de la misma, disposiciones relativas al cuerpo de inspectores por ejemplo o el procedimiento específico para llevar a cabo la inspección. Así mismo lo relativo a las formas de requerir la inspección, ni los sujetos que pueden solicitarla. Sin 
dudas retos normativos a los cuales se enfrenta la inspección del trabajo en Cuba.

El Decreto 100 por su parte, regula los particulares de todas las inspecciones estatales. Un ejemplo, es que salva el vacío relativo a los inspectores. El Capítulo II de dicho cuerpo legal establece los sujetos que pueden efectuar las inspecciones estatales y los define: inspectores profesionales, eventuales y populares.

Así mismo establece los requisitos formales para ser inspector:

a. mantener una conducta política y moral dentro de los principios y objetivos de nuestro Estado Socialista;

b. ser graduado de nivel medio superior como mínimo, y poseer, además, los conocimientos específicos que se requieren en cuanto a la actividad inspeccionar;

c. los demás requisitos específicos que exija el mismo o la dependencia administrativa del órgano correspondiente, los cuales determinarán también las condiciones adicionales que se exigirán a los inspectores principales o jefes de grupo.

Igualmente, quedan establecidos los deberes y facultades de los inspectores en sus ámbitos de actuación.

Nuevos retos se vislumbran. El ordenamiento jurídico actual no establece como ámbito de inspección a los establecimientos industriales -según el Convenio 81 ratificado-, y trata de saldar esta deuda supeditando la inspección a lo que la ley de trabajo identifica como empleadores ${ }^{25}$. Así, tanto en las formas organizativas de la inversión extranjera, que se rigen por la legislación laboral vigente ${ }^{26}$, como en las cooperativas y otro tipo de asociaciones como las organizaciones no gubernamentales (ONGs), se hace difícil la implementación de la inspección del trabajo en

\footnotetext{
25 Cfr. Artículo 192 del vigente CT.

26 Cfr. Artículo 2 de la Resolución No. 16 de 14 de abril de 2014 del Ministerio de Trabajo y Seguridad Social sobre el régimen laboral en la inversión extranjera en Gaceta Oficial Extraordinaria No. 20 de 16 de abril de 2014.
} 
tanto el procedimiento efectuado es aún, "deficiente». Lo cierto es que la inspección del trabajo debe estar presente donde quiera que existan relaciones jurídicas laborales, sin distinción entre lo público y lo privado.

Siendo que la inspección del trabajo en Cuba tiene como fin el de controlar el cumplimiento de la legislación laboral y de seguridad social ${ }^{27}$; su articulación jurídica se convierte en un reto pues olvida el ámbito de actuación que se circunscribe a las relaciones laborales. En otro sentido, la inspección laboral no tiene alcance en las relaciones jurídicas de los llamados cuadros y funcionarios; sino solo en relación con las relaciones laborales establecidas.

También otro reto que deriva de la regulación actual es la identificación de las infracciones cometidas por el empleador. El CT dispone -por materias- los elementos sobre los cuales incidirá la inspección ${ }^{28}$. Ello hace necesario un análisis, precisamente porque se desborda de materias que no le corresponden. Igualmente, añade al articulado un "artículo saco» que en buena lid es el que define el ámbito de actuación de la inspección ${ }^{29}$.

A la par, la realidad laboral cubana muestra nuevos derroteros para la inspección del trabajo. Al ser una de las principales consecuencias del redimensionamiento de las relaciones laborales, la aparición de nuevos empleadores como sujetos del Derecho del Trabajo y, hasta este instante la inspección del trabajo erigirse sobre un andamiaje del Estado para controlarse a sí mismo como empleador, este nuevo contexto conspira contra su correcta ejecución en los establecimientos del sector privado.

A ello se suma la realidad económica que vive el país que incide en los bajos salarios en el sector público y en el traslado hacia el sector privado de trabajadores que a cambio de

27 Cfr. Artículo 190 del CT.

28 Cfr. Arts. 224 y ss. del Decreto No. 326 Reglamento del Código de Trabajo.

29 Cfr. Art. 230, incisos a) y b) del Decreto No. 326 Reglamento del Código de Trabajo. 
una mejor remuneración permiten la violación de sus derechos laborales.

Sin embargo, los controles en los establecimientos del sector privado se limitan a la inspección sanitaria, a constatar la existencia de un contrato laboral o de la licencia para el ejercicio del trabajo por cuenta propia, pero en muy pocas ocasiones se realizan para verificar, por ejemplo, lo relativo al cumplimiento de la jornada de trabajo de 8 horas. De esta forma, los trabajadores subordinados del sector privado requieren del buen funcionamiento de la inspección para consagrar el respeto hacia sus derechos laborales.

Por su parte, en el sector estatal, donde se supone están bien definidos los elementos de la inspección, no son tampoco eficientes los controles. Se encuentran vinculados a las inspecciones estatales generales negando su independencia $\mathrm{y}$ autonomía. Así, se limita el control, básicamente, a la investigación de los sucesos acaecidos que provocaron un accidente de trabajo.

A todo esto, se suman las deficiencias normativas. Si bien está ordenada, aún carece de sistematización, comenzando por el hecho de que no posee un cuerpo orgánico especial, sino que confluye su regulación, más estructurada y condensada, entre el vigente CT y su Reglamento con las "especificidades» de los Decretos No. 100 y 116 que fueron promulgados en realidades desfasadas. Se observa así la incorrecta percepción de la inspección del trabajo lo que hace que corra igual suerte su ordenación jurídica y causa que deje de cumplir sus funciones tuitiva, preventiva y de control, convirtiéndose en un mecanismo de represión.

Lo cierto es que la inspección del trabajo vislumbra nuevos desafíos a partir de las deficiencias e insuficiencias que muestra la falta de sistematización y coherencia del ordenamiento jurídico laboral ante las nuevas realidades. Sin embargo, es dable reconocer la participación de Cuba como miembro de la OIT, donde desde su fundación ratificó el Convenio 81 y 
en su ordenamiento jurídico laboral se muestra claramente la intención del Estado por el reconocimiento de los derechos y garantías a los trabajadores. Sobre todo, la inspección del trabajo como mecanismo de control es un elemento trascendental del ordenamiento laboral cubano.

\section{Referencias}

Abramovich, V. y Courtis, Ch. (s.f). Futuros posibles. El Derecho Laboral en la encrucijada. Revista Jurídica de la Universidad de Palermo.

Alonso Olea, M. (1981). Curso de Derecho del Trabajo. Barcelona/ Caracas/México: Editorial Ariel.

Alonso Olea, M. y Casas Baamonde, M. E. (1995) Derecho del Trabajo. Madrid: Universidad de Madrid.

Blasco Pellicer, Á. y García Rubio, Ma. A. (2001). Curso de Derecho Administrativo Laboral. Valencia: Editorial Tirant lo Blanch.

Bermejo Vera, J. (1998). La administración inspectora. Revista de Administración Pública. Madrid: Centro de Estudios Políticos y Constitucionales. 147 (septiembre-diciembre).

Cano Campo, Tomás. (2013). La presunción de validez de las actas de inspección. Actas del vIII Congreso de la Asociación Española de Profesores de Derecho Administrativo. Madrid, España: INAP.

Casale, G. y Fasani, M. (2013). Nuevos desafíos para la administración e inspección del trabajo en el contexto de la reciente crisis económica. Revista Latinoamericana de Derecho Social. No. 17 (julio-diciembre).

De Aguinaga Tellería, A. (1952). Derecho del Trabajo. Madrid, España: Gráficas González.

De Buen, N. (1984). Derecho del Trabajo. México: Editorial Porrúa S. A.

Diéguez, G. (1995). Lecciones de Derecho del Trabajo. Madrid, España: Editorial Marcial Pons.

García Costa, Francisco Manuel. (2011). Revista Documentación Administrativa. Madrid, España: Instituto Nacional de Administración Pública 289 (enero-abril). 
Grisola, Julio. (1999) Derecho del Trabajo y la Seguridad Social. Editorial Depalma.

Hernainz Márquez, M. (1951). Tratado elemental de Derecho del Trabajo. Madrid, España: Instituto de Estudios Políticos.

Matilla Correa, Andry. (2017). Cinco principios jurídico-administrativos en clave teórica de buena administración: eficacia, eficiencia, objetividad, economía o economicidad y celeridad. Andry Matilla Correa, (Coord.) Tendencias Actuales del Derecho Administrativo. Homenaje al profesor Dr. Héctor J. Garcini Guerra. La Habana, Cuba: uniJuRIs.

Míguez Macho, Luis. (2011). El principio de objetividad en el procedimiento administrativo. Revista Documentación Administrativa. 289 (enero-abril). Madrid, España: Instituto Nacional de Administración Pública, 119 y ss.

Leal Vásquez, Brigitte. (2015). Planificación, colaboración y autocontrol: nuevos instrumentos normativos para la administración fiscalizadora. Revista de Derecho Público. 83 (2).

OIT. (2011). Estudio sobre infracciones y sanciones en el ámbito de la inspección del trabajo: el caso de Costa Rica, El Salvador, Guatemala, Honduras, Nicaragua y República Dominicana, Ginebra, disponible en www.ilo.org/public/libdoc/ilo/2011/ 154818_span.pdf, consultado el 27 de noviembre de 2017.

OIT. (2010). La inspección del trabajo en Europa: trabajo no declarado, migración y tráfico de personas, Ginebra, disponible en http://www.ilo.org/wcmsp5/groups/public/---ed_dialogue/--lab_admin/documents/publication/wcms_144915.pdf, consultado el 30 de marzo de 2017.

OIT. (2009). Programa sobre Administración e Inspección del Trabajo. Administración del Trabajo: Asegurar la gobernanza a través de la aplicación de la ley en América Latina. El papel central de la inspección del trabajo, Ginebra, disponible en http://www.ilo.org/wcmsp5/groups/public/@ed_dialogue/@ lab_admin/documents/publication/wcms_116047.pdf, consultado el 30 de marzo de 2017.

Pig, P. (1922). Traité élémentaire de législation industrielle. Les lois ouvrières. París, Francia: Arthur Rousseau Éditeur.

Rivero, Jean. (1990). Droit Administratif. París, Francia: Jurisprudence Genérale Dalloz. 
Sánchez Ramírez, Amed y Antúnez Sánchez, Alcides. (2018). El régimen jurídico de la inspección administrativa y los derechos sociales en Cuba. Apuntes para una reforma. Revista Jurídica Direito\& Paz, Direito\& Paz. X (38).

Stiftung, Konrad Adenauer. (2003). Jurisprudencia del Tribunal Constitucional Federal alemán, JürgenSchwabe, comp. de sentencias, Marcela Anzola-Gil, trad., Konrad Adenauer Stiftung, México.

Vázquez Vialard, Antonio. (1999). Derecho del Trabajo y de la Seguridad Social. Buenos Aires, Argentina: Editorial Astrea.

Viamontes Guilbeaux, Eulalia. (2007). Derecho Laboral Cubano. Teoría y Legislación. La Habana, Cuba: Félix Varela. 